\title{
An Implementation of Hierarchical Signal Processing on Wireless Sensor in TinyOS Environment
}

\author{
Chris Otto, John P. Gober, Reggie W. McMurtrey, Aleksandar Milenković, Emil Jovanov \\ Electrical and Computer Engineering Dept. \\ The University of Alabama in Huntsville \\ jovanov@ece.uah.edu
}

\begin{abstract}
Wireless intelligent sensor networks have become a major infrastructure for deeply embedded systems. As these systems typically operate under stringent energy constraints, a careful consideration of computation and communication is crucial for extended system lifetime. To achieve extended lifetime on a limited energy budget the existing sensor platforms feature limited communication bandwidth, processing power, and interfacing capabilities. We propose to augment a general-purpose sensor platform by low-power, intelligent signal processing modules. This approach allows on-sensor parallel signal processing. In this paper we present an implementation of a sensor node featuring 2-tier hierarchical organization. The upper level is realized using the Telos wireless platform, and the lower level is a custom Intelligent Signal Processing Module (ISPM). The ISPM is developed as an application-specific sensor board for health monitoring applications. It features two accelerometers, a bioamplifier, and a microcontroller used for preprocessing and communication with the Telos platform. Telos features ZigBee wireless interface and can communicate to an application server through a network coordinator. The application server represents a third tier in the hierarchical system. The server seamlessly integrates sensors into a wireless body area network of physiological sensors. We describe the system architecture and software support implemented in TinyOS environment.
\end{abstract}

\section{Keywords}

Wireless sensor networks, Hierarchical signal processing, Activity monitor, ECG monitor.

\section{INTRODUCTION}

Wireless sensor networks with intelligent nodes deeply embedded into the physical environment have recently emerged as a new tier in the information technology [1]. They promise a wide range of new applications, such as habitat monitoring, machine health monitoring and guidance, traffic pattern monitoring and navigation, plant monitoring in agriculture, and infrastructure monitoring. One of the most promising domains is health monitoring.

Permission to make digital or hard copies of all or part of this work for personal or classroom use is granted without fee provided that copies are not made or distributed for profit or commercial advantage and that copies bear this notice and the full citation on the first page. To copy otherwise, or republish, to post on servers or to redistribute to lists, requires prior specific permission and/or a fee.

$43^{\text {rd }}$ ACM Southeast Conference, March 18-20, 2005, Kennesaw, GA, USA. Copyright 2005 ACM 1-59593-059-0/05/0003 ...\$5.00.
A number of physiological sensors, such as motion sensors, electrocardiogram (ECG), electromyogram (EMG), or electroencephalogram (EEG) can be seamlessly integrated into a wearable Wireless Body Area Network (WBAN). The WBAN consists of inexpensive, lightweight, and miniature physiological sensors, and allows prolonged, unobtrusive health monitoring, computer-supervised rehabilitation, and even early detection of medical conditions [2][3][4]. For example, intelligent heart monitors can warn the user about impeding medical conditions [6] or provide information for a specialized service in the case of catastrophic events [7]. Monitoring of user's physical activity is significant application of mobile ambulatory monitoring during process of physical rehabilitation [8][9].

Our target application is monitoring of physical activity using WBAN of intelligent motion and ECG sensors [2]. Motion sensors can be deployed on characteristic body points, such as ankle, chest, wrist, and hip. Real time analysis of signals from motion sensors can provide information about the user's position and state of activity (laying, sitting, walking, running).

Design of sensor nodes optimized for sensor-specific signal processing and ultra-low power consumption poses one of the most challenging tasks. New application framework requires careful system design due to prolonged autonomous sensor operation and limited battery power. Hierarchical system organization has been proposed as a solution for energy-efficient sensor networks [5].

We propose to augment a general-purpose sensor platform by low-power, intelligent signal processing modules. This approach has the following advantages: a) increased processing power due to parallel execution, b) capability to interface multiple physical sensors on a single network node, and c) off-the-shelf sensor platforms provide low-price and system support.

We employ a 2-tier hierarchical organization for each network node. The lower level is a custom Intelligent Signal Processing Module (ISPM). The ISPM is developed as an application-specific sensor board with an accelerometer based motion sensor and a bioamplifier. An ultra low power microcontroller MSP430F1232 is used for preprocessing and communication with the upper level. The Telos platform is used as the second tier. Telos features ZigBee wireless interface and can communicate to an application server through a network coordinator. The application server represents a third tier in the hierarchical system, and can be implemented on a PDA, personal computer, or a cell-phone. The server is used to set up the WBAN, configure sensors, control and collect data from sensor nodes. Although the proposed system is tailored for health monitoring applications, this approach will benefit other wireless sensor network applications.

The following section describes the system architecture and a custom developed ISPM module. Section three outlines software organization in TinyOS environment, and section four concludes the paper. 


\section{SYSTEM ARCHITECTURE}

General architecture of the proposed wireless network is illustrated in Figure 1. The wireless network consists of a number of sensor platforms that communicate with a network coordinator using ZigBee wireless interface. The network coordinator is another Telos platform attached to a PDA or a PC, which serves as an application server.

A sensor platform typically incorporates two levels of hierarchy. The upper level is realized using the Telos wireless platform, and the lower level is a sensor-specific intelligent signalprocessing module.

For the tier two processing board, we used a commercially available wireless sensor platform Telos from Moteiv [10]. The Telos platform is an ideal fit for this application due to small footprint and TinyOS support [11]. Each Telos board is based on an $8 \mathrm{MHz}$ MSP430F149 microcontroller with integrated $2 \mathrm{~KB}$ of RAM and $60 \mathrm{~KB}$ of flash memory, a USB interface, and an integrated wireless ZigBee radio with antenna [12]. In addition, the Telos platform includes humidity, temperature, and light sensors that might be of interest for some applications.

Telos platform features a 10-pin expansion connector that allows one UART and I2C interface, two general-purpose I/O lines, and three analog inputs.

The tier one of the hierarchical system is implemented as the Intelligent Signal Processing Module (ISPM), designed to interconnect with the Telos wireless sensor platform as tier two. Block diagram of the sensor node is shown in Figure 2 and picture of the node and ISPM module is given in Figure 3.

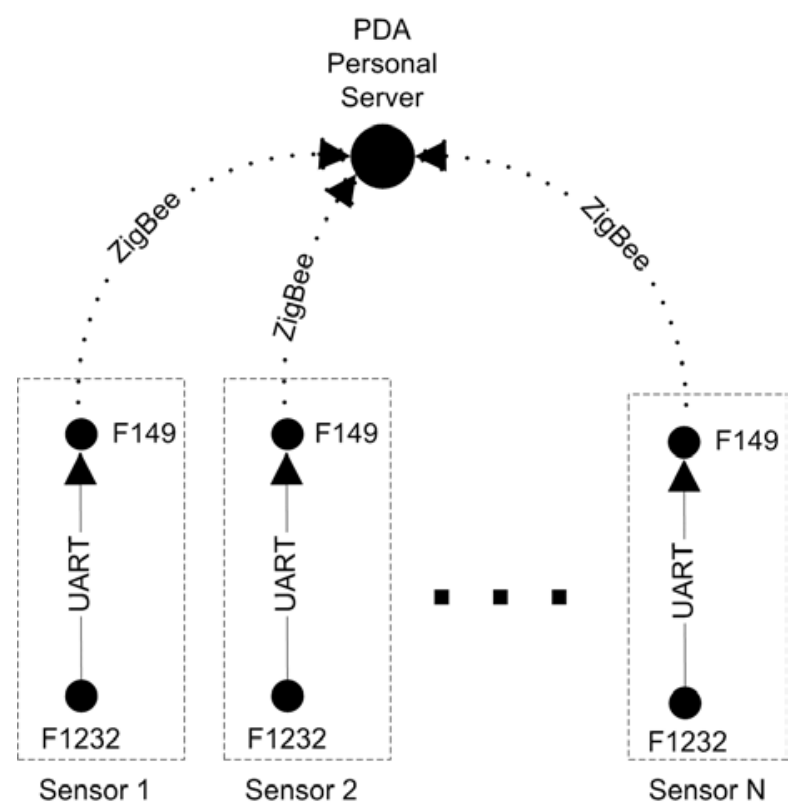

Figure 1 Hierarchical system organization
The ISPM extends the capabilities of Telos by adding two perpendicular dual axis accelerometers (Analog Devices ADXL202), a bioamplifier with signal conditioning circuit, and a microcontroller MSP430F1232. The ISPM's two ADXL202 accelerometers cover all three axes of motion. One ADXL202 is mounted directly on the ISPM board and collects data for the X and Y axes. The second ADXL202 is mounted on a daughter card from SparkFun [13] that extends vertically from the ISPM (Connector J3 on Figure 3a). This accelerometer collects acceleration data on the $Z$ axis.

The user's physiological state is monitored using on-board bioamplifier implemented using instrumentation amplifier (INA321) with signal conditioning circuit. The bioamplifier could be used for electromyogram (EMG) or electrocardiogram (ECG) monitoring. The output of the signal conditioning circuit is connected to the local microcontroller as well as to the microcontroller on the Telos board via the expansion connector. The MSP430F149 ADC on the Telos board has higher resolution (12 bit) than the MSP430F1232 on ISPM. This configuration gives the user the added flexibility of utilizing either microcontroller to process physiological signals.

The ISPM has its own MSP430F1232 processor for sampling and low-level-data processing. An alternative is to use a signal condition circuit and use Telos' microcontroller for data acquisition and processing [7]. This microcontroller was selected primarily for its compact size and excellent MIPS/mW ratio. Therefore, an additional microcontroller improves the processing power of a sensor node with no significant overhead in power consumption $(<1 \mathrm{~mW})$. Other features that were desirable for this design were the 10-bit ADC and the timer capture/compare registers that are used for acquisition of data from the accelerometers. The F1232 also has hardware UART that is used for communications with the Telos board.

The ISPM features four layer PCB and measures $1.25 " \mathrm{x}$ 1.57'. It was designed to fit within the outline of the Telos board. The ISPM board does not extend over Telos' integrated PCB antenna. To reduce the noise from the digital components on the analog signal conditioning circuit, the analog and digital circuits were placed on opposite sides of the board. Additionally, the 4layer design allowed placement of a ground plane underneath the ECG circuit to further reduce digital/wireless noise.

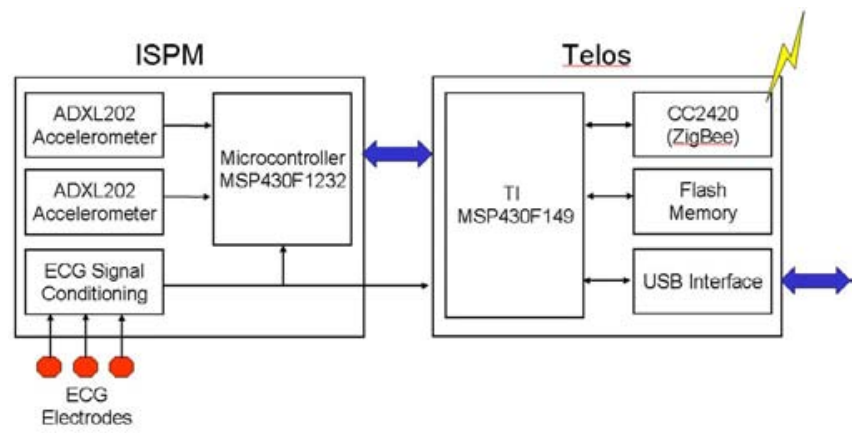

Figure 2 Block diagram of the sensor node 

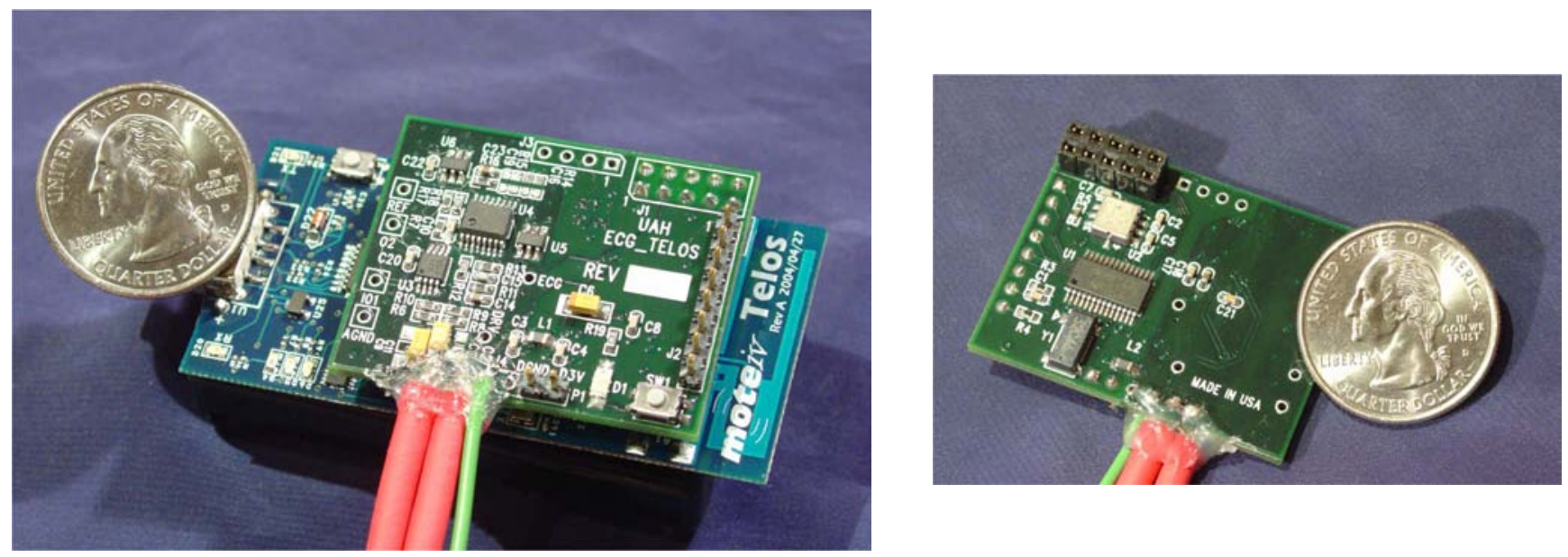

Figure 3 Telos board with ISPM module

\section{SOFTWARE ORGANIZATION}

A typical wireless sensor network system includes a network of wireless nodes where each node represents the first two tiers of the hierarchical system. The ISPM, being the lowest tier (tier one), interfaces directly with the sensors for data retrieval and also has enough data processing bandwidth for preliminary signal processing (pre-processing). The pre-processed data is then transferred to the tier two Telos board. Tier two processing includes more sophisticated real-time analysis and is capable of recognizing significant patterns of data, characterization, additional filtering, etc. The ISPM and the Telos board are organized in a master-slave relationship. Telos board controls delivery of data and type of processing performed on the ISPM module via the UART serial interface. Depending on the application and the intensity of signal processing requirements, the Telos board is also capable of transmitting data wirelessly to a top tier (tier three) signalprocessing node such as a wirelessly enabled PDA or desktop computer (Figure 1).

The software system is implemented in TinyOS environment [11]. The TinyOS operating system provides an extremely lightweight, open source operating system for wireless embedded sensors. It is designed to use minimal resources and its configuration is defined at compile time rather than runtime. This allows applications to select functionality by choosing components from the TinyOS component library or writing their own components. This approach results in a natural modular design, minimal use of resources, and short development cycles.

TinyOS application is implemented as a set of component modules written in nesC [14]. The nesC language extends the $\mathrm{C}$ language with new constructs to facilitate the component architecture and multitasking. By adding direct language support for synchronization and task management, it allows rapid development and minimizes resources.

\subsection{ISPM Software}

The ISPM board handles acquisition of accelerometer data and preprocesses the signals. In our current implementation, the ISPM samples three independent accelerometer axes each at a rate of $200 \mathrm{~Hz}$. The raw accelerometer data is filtered and preprocessed.
Typical raw signal after filtering is shown in Figure 4. The filtering includes a moving average filter to eliminate high frequency movement artifacts, and separation of low and high frequency components of the acceleration signal.

Sensor orientation can be calculated as angle between low frequency components. Activity estimation and force detection utilize AC components. User activity is correlated with a function based on the sum of integrals of AC components in each channel [8].

\subsection{Telos Software}

The Telos module is the master controller on each node. It requests data from the ISPM module every $25 \mathrm{~ms}$. The ISPM board is restricted from sending any unsolicited data to the Telos board. Information is requested by raising interrupt line or sending request for data from the Telos board. The data set received from the ISPM module is additionally processed and analyzed. The Telos board is responsible for wireless communications and transporting data to other peer sensors or to a network coordinator. The processed data set can be stored in an external serial flash memory in the case of autonomous operation or if the wireless channel is not available.

The Telos application software is implemented as multiple TinyOS components encompassing the following high-level functions: wireless communications, extended flash storage, messaging software, board-to-board communications, and tier two signal processing.

The Telos board and ISPM module are connected via an asynchronous serial interface. A simple communication protocol is employed to eliminate any protocol overhead on either end. The Telos processor sends a single byte command to request data. The upper three bits are used for command type and the remaining five bits are reserved for application specific argument passing. For ISPM responses, the request-reply protocol identifies the start of a frame, the next byte is an echo of the Telos generated command, which identifies the response type and serves as an acknowledgement.

The Telos extension header provides only UART0 interface. (USART0 on the Telos' processor). The main problem we faced in system design is sharing of USART0 between the ISPM module, the serial flash, and the wireless controller. Therefore, the 


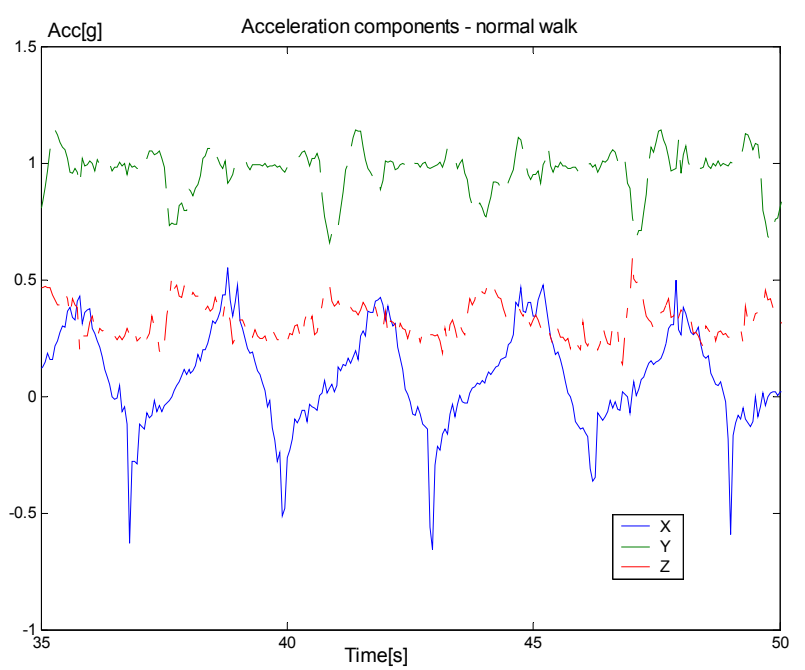

Figure 4 Real time display of raw accelerometer signals taken from the right leg during ten steps of normal walk

processor can communicate either with the ISPM board or the wireless radio/serial flash. The external serial flash and wireless radio chipset are connected via the Synchronous SPI interface, which also uses USART0. Current TinyOS component, BusArbitration, addresses the sharing of the SPI interface between the multiple external peripherals; however, it does not address multiplexing between asynchronous and synchronous operation. Typically, this USART would be statically initialized to one function; however, this implementation requires a time-sharing of the hardware resource and dynamic selection of USART protocol for each sampling interval ( $25 \mathrm{~ms}$ in the current implementation).

It should be noted that when switching USART functionality, the HPLUSARTFeedback.txDone() event is not sufficient indication that the USART has completed the transmission. For the MSP430 platform this event is signaled in response to the UART transmit interrupt handler - indicating that the UxTXBUF has been transferred to the UART transmit shift register. At the time of notification, the last byte transmission has just begun. This enables an application to transfer the next byte before current transmission stops. One character time remains before the USART has completed communications. For this reason, our implementation requires an additional timeout of one character time before calling the BusArbitration releaseBus() command

In the current software implementation, we pushed control of the BusArbitration interface into a separate module, and control the arbitration at the application layer per-frame by utilizing SplitControl interface. This implementation allows all existing applications and TinyOS components to remain intact. They can continue to interface with BusArbitration using the existing interface.

\section{CONCLUSION}

In this paper we described hardware and software support for a hierarchical processing on wireless sensor in TinyOS environment. The hierarchical signal processing allows system optimization by balancing the processing load between the different levels of hierarchy. In our application, the F1232 on the ISPM can be used to sample data and transmit it to Telos, or if the application requires maximum processing power, the F1232 can be used to augment the processing capabilities of the Telos' F149 microcontroller.

The current implementation targets characterization and quantification of user's activity. Accelerometer and physiological data is sampled and preprocessed at the ISPM module. The result is then transmitted to the Telos board for additional processing, flash memory storage, and context-specific actions taken based on data interpretation. The third tier, personal PDA server, integrates information from individual sensors and provides a synergy of information.

We plan to perform real-time power profiling and optimize task partitioning between two tiers. The optimized processing will maximize the battery life.

\section{ACKNOWLEDGMENTS}

This work is being supported in part by National Science Foundation grant IIS-0434156. We would like to thank Mr. Forrest Arndt and Advanced Circuits Inc. for their support in PCB manufacturing.

\section{REFERENCES}

[1] Estrin, D., Culler D., Pister, K., Sukhatme, G. Connecting the Physical World with Pervasive Networks. IEEE Pervasive Computing, Volume 1, Issue 1, (Jan. 2002), 59-69.

[2] Jovanov, E., Price, J., Raskovic, D., Kavi, K., Martin, T., Adhami, R. Wireless Personal Area Networks in Telemedical Environment. In Proceddings of the Third International Conference on Information technology in Biomedicine (ITAB-ITIS2000), (Arlington, VA), 2000, 22-27.

[3] Wearable Technology, Special Issue of the IEEE Engineering in Medicine and Biology Magazine, 22, 3, (May/June 2003).

[4] Jovanov, E., Milenkovic, A., Basham, S., Clark, D., Kelley, D. Reconfigurable Intelligent Sensors for Health Monitoring: A Case Study of Pulse Oximeter Sensor. In Proceedings of the 26th Annual International Conference of the IEEE Engineering in Medicine and Biology Society, (San Francisco, CA, September 2004), 4759-4762.

[5] Raskovic, D., Energy-Efficient Hierarchical Processing In The Network Of Wireless Intelligent Sensors (WISE). $\mathrm{Ph}$.D. thesis, University of Alabama in Huntsville, Huntsville, AL, 2003.

[6] Welch, J., Guilak, F., Baker, S.D. A Wireless ECG Smart Sensor for Broad Application in Life Threatening Event Detection. In Proceedings of the 26th Annual International Conference of the IEEE Engineering in Medicine and Biology Society, (San Francisco, CA, September 2004), 3447-3449.

[7] Malan, D., Fulford-Jones, T.R.F., Welsh, M., Moulton, S. CodeBlue: An Ad Hoc Sensor Network Infrastructure for Emergency Medical Care. In Proc. of the MobiSys 2004 Workshop on Applications of Mobile Embedded Systems (WAMES 2004). (Boston, MA, June, 2004), 1214. 
[8] Mathie, M.J., Celler, B.G. A System For Monitoring Posture And Physical Activity Using Accelerometers. In Proc. of the 23rd Annual International Conference of the IEEE Engineering in Medicine and Biology Society, 2001, 3654- 3657. .

[9] Lee, S.W., Mase, K. Activity and Location Recognition Using Wearable Sensors. Pervasive Computing, JulySeptember 2002, Vol. 1, No. 3, pp. 24-32.

[10] Moteiv, http://www.moteiv.com
[11] TinyOS, http://www.tinyos.net

[12] ZigBee Alliance, http://www.zigbee.org/

[13] Spark Fun Electronics, http://www.sparkfun.com/

[14] Gay. D., Levis, P., von Behren, R., Welsh, M., Brewer, E., Culler, D.The nesC Language: A Holistic Approach to Networked Embedded Systems, in Proceedings of Programming Language Design and Implementation (PLDI) 2003, June 2003. 